\title{
Composition and Variability of Epicuticular Lipids of Azaleas and their Relationship to Azalea Lace Bug Resistance
}

\author{
Yuefang Wang, ${ }^{1}$ S. Kristine Braman, ${ }^{2,3}$ Carol D. Robacker, ${ }^{1}$ and Joyce G. Latimer ${ }^{1}$ \\ The University of Georgia, Georgia Station, Griffin, GA 30223 \\ Karl E. Espelie \\ Department of Entomology, The University of Georgia, Athens, GA 30602
} AdDitional INDEX words. Stephanitis pyrioides (Scott), Rhododendron sp., host plant resistance, $\alpha$-amyrin, $\beta$-amyrin, ursolic
acid, $n$-hentriacontane

\begin{abstract}
Epicuticular lipids were extracted from the foliage of six deciduous and one evergreen azalea genotypes (Rhododendron sp.) and identified by gas chromatography-mass spectrometry. The relationship of leaf-surface lipid composition with measures of resistance to azalea lace bug, Stephanitis pyrioides Scott, was evaluated. Each genotype had a distinct epicuticular lipid composition. The major surface lipid components from all test taxa were $n$-alkanes and triterpenoids. In the most resistant genotypes [R. canescens Michaux and $R$. periclymenoides (Michaux) Shinners] ursolic acid, $n$-hentriacontane, and $n$-nonacosane were the most abundant epicuticular lipids. The lipids present in largest proportion among all susceptible deciduous genotypes tested were $\alpha$-amyrin, $\beta$-amyrin, and $n$-nonacosane. The proportions of the lipid components from the same plant of each genotype varied between spring and fall samples. Among classes of lipids, $\boldsymbol{n}$-alkanes, $\boldsymbol{n}$-1-alkanols, and triterpenoids had significant correlations with azalea lace bug behavior on host plants. Among individual components, heptadecanoic acid, $n$-hentriacontane, oleanolic acid, ursolic acid and one unknown compound (with major mass spectra 73/179/192/284/311) were significantly negatively correlated with host plant susceptibility to azalea lace bug, as measured by oviposition, leaf area damaged, egg and nymphal development, and nymphal survivorship. Triacontanol, $\alpha$-amyrin, $\beta$-amyrin, and three unknowns were significantly positively correlated with host plant susceptibility. Acceptance or rejection by azalea lace bug to a particular plant may be mediated by a balance of positively and negatively interpreted sensory signals evoked by plant chemicals. This study indicated that the high levels of resistance observed in $R$. canescens and $R$. periclymenoides may be due to the lesser amount or the absence of attractants and stimulants for feeding or oviposition.
\end{abstract}

Azalea lace bug, Stephanitis pyrioides Scott (Heteroptera: Tingidae), is a key pest of azaleas in the United States (Braman et al., 1992; Neal and Douglass, 1988; Raupp and Noland, 1984). Researchers have recently identified high levels of resistance to azalea lace bug among deciduous azaleas (Rhododendron sp.) (Braman and Pendley, 1992; Wang etal., 1998). Understanding the basis of azalealace bug resistance can facilitate breeding new resistant cultivars.

Although the primary function of plant epicuticular lipids on aerial plant surfaces is prevention of water loss, they also function ecologically in the mediation of interactions between plants and their insect herbivores (Eigenbrode and Espelie, 1995). Here we determine the leaf-surface lipid composition of resistant and susceptible azalea genotypes. The composition of epicuticular lipids of winter wheat (Triticum aestivum L.) was correlated with resistance to the English grain aphid, Sitobion avenae F. (Lowe et al., 1985). Resistance in sorghum, Sorghum bicolor L., to the green bug, Schizaphis graminum Rondani, has been correlated with amounts of epicuticular lipids on leaves (Starks and Weibel, 1981). High levels of $n$ alkanols contribute to insect resistance, such as docosanol in tobacco (Nicotiana tabacum L.) resistant to the tobacco budworm, Heliothis

Received for publication 14 Aug. 1998. Accepted for publication 18 Feb. 1999. We thank S.A. Baden, University of Georgia, for her assistance with GC-MS and Yongfu Ge for his assistance with data processing. We thank B.R. Wiseman for critically reviewing the manuscript. The research reported herein is a portion of a thesis submitted by Y.W. in partial fulfillment of the requirements for the MS degree. The cost of publishing this paper was defrayed in part by the payment of page charges. Under postal regulations, this paper therefore must be hereby marked advertisement solely to indicate this fact.

${ }^{1}$ Department of Horticulture.

${ }^{2}$ Department of Entomology.

${ }^{3}$ To whom reprint requests should be addressed. virescens Fab. (Johnson and Severson, 1984) and triacontanol in alfalfa (Medicago sativa L.) resistant to the spotted alfalfa aphid, Therioaphis maculata Buckton (Bergman et al. 1991). Some triterpenoids have also been known to function in insect resistance. High levels of $\beta$-amyrin were associated with resistance of raspberry cultivars (Rubus sp.) to the raspberry aphid, Amphorophora idaei Börner (Robertson et al. 1991).

Preference for certain azalea genotypes by azalea lace bug may be influenced by some epicuticular lipid compounds from the foliage (Balsdon et al. 1995). In the present study, the epicuticular lipids of foliage from seven azalea taxa were analyzed by gas chromatography-mass spectrometry to determine if differences exist among plants expressing varying degrees of resistance to azalea lace bug. The relationship of the classes of compounds and certain components of epicuticular lipids to observed azalea lace bug resistance was evaluated.

\section{Materials and Methods}

Plant materials. Laboratory and field bioassays characterized the resistance of deciduous azalea taxa (Wang et al., 1998). Antibiosis was suggested by lower survivorship of lace bugs and reduced oviposition. Seven taxa representing a range of resistance levels were selected for further study. The resistant taxa evaluated were the deciduous R. canescens Michaux and R.periclymenoides (Michaux) Shinners. Susceptible taxa included 'My Mary', a complex hybrid with $R$. atlanticum Ashe, $R$. periclymenoides, and $R$. austrinum (Small) Rehder in the parentage; two genotypes of $R$. viscosum (L.) Torrey, R. atlanticum; and one evergreen cultivar, 'Delaware Valley White', which is a selection of $R$. indica var. alba. One $R$. viscosum 
Table 1. Mean percentage $( \pm \mathrm{SD})$ of epicuticular lipids extracted from fall and spring leaves of seven azalea genotypes. ${ }^{\mathrm{z}}$

\begin{tabular}{|c|c|c|c|c|c|c|c|}
\hline \multirow[b]{2}{*}{ Peak } & \multirow{2}{*}{$\begin{array}{l}\text { Retention } \\
\text { time } \\
\text { component }^{\mathrm{y}}\end{array}$} & \multicolumn{2}{|c|}{$\begin{array}{c}\text { Delaware } \\
\text { Valley White } \\
\text { (susceptible) }\end{array}$} & \multicolumn{2}{|c|}{$\begin{array}{c}\text { My Mary } \\
\text { (susceptible) }\end{array}$} & \multicolumn{2}{|c|}{$\begin{array}{c}R . \\
\text { serrulatum } \\
\text { (susceptible) }\end{array}$} \\
\hline & & Fall & Spring & Fall & Spring & Fall & Spring \\
\hline$\overline{1}$ & 17.80 Hexadecanoic acid & $0.4 \pm 0.1$ & $0.9 \pm 1.1$ & $0.4 \pm 0.0$ & $0.7 \pm 0.2$ & $0.3 \pm 0.2$ & $1.4 \pm 1.2$ \\
\hline 2 & 18.23 Unk 55/91/119/309 & $0.2 \pm 0.2$ & $<0.1$ & $0.0 \pm 0.0$ & $0.3 \pm 0.1$ & $0.0 \pm 0.0$ & $0.6 \pm 1.1$ \\
\hline 3 & 18.46 Heptadecanoic acid & $0.1 \pm 0.1$ & $<0.1$ & $0.0 \pm 0.0$ & $0.2 \pm 0.1$ & $0.0 \pm 0.0$ & $0.4 \pm 0.4$ \\
\hline 4 & $18.83 n$-Docosane & $0.1 \pm 0.1$ & $0.0 \pm 0.0$ & $0.0 \pm 0.0$ & $0.1 \pm 0.1$ & $0.1 \pm 0.1$ & $0.3 \pm 0.4$ \\
\hline 5 & 18.95 Octadecenoic acid & $0.3 \pm 0.2$ & $0.9 \pm 0.8$ & $0.5 \pm 0.1$ & $0.8 \pm 0.2$ & $0.3 \pm 0.3$ & $1.4 \pm 1.2$ \\
\hline 6 & 19.09 Octadecanoic acid & $0.2 \pm 0.1$ & $0.6 \pm 1.0$ & $0.5 \pm 0.1$ & $0.6 \pm 0.2$ & $0.1 \pm 0.1$ & $0.7 \pm 1.0$ \\
\hline 7 & $19.43 n$-Tricosane & $0.2 \pm 0.2$ & $0.1 \pm 0.1$ & $0.2 \pm 0.1$ & $0.3 \pm 0.2$ & $0.2 \pm 0.2$ & $0.6 \pm 0.8$ \\
\hline 8 & $20.03 n$-Tetracosane & $1.1 \pm 1.7$ & $0.4 \pm 0.2$ & $0.6 \pm 0.2$ & $0.5 \pm 0.1$ & $0.3 \pm 0.2$ & $0.9 \pm 1.3$ \\
\hline 9 & 20.24 Eicosanoic acid & $0.5 \pm 0.3$ & $0.5 \pm 0.3$ & $0.8 \pm 0.2$ & $0.9 \pm 0.3$ & $0.7 \pm 0.5$ & $1.8 \pm 1.7$ \\
\hline 10 & $20.62 n$-Pentacosane & $0.3 \pm 0.2$ & $0.5 \pm 0.2$ & $0.5 \pm 0.1$ & $1.1 \pm 0.3$ & $0.3 \pm 0.1$ & $0.7 \pm 0.9$ \\
\hline 11 & 20.99 Unk 73/119/387/402 & $0.3 \pm 0.3$ & $0.3 \pm 0.3$ & $0.3 \pm 0.2$ & $0.2 \pm 0.1$ & $0.3 \pm 0.2$ & $0.8 \pm 1.1$ \\
\hline 12 & 21.04 Unk 57/149/279 & $0.1 \pm 0.1$ & $0.3 \pm 0.4$ & $0.1 \pm 0.2$ & $0.3 \pm 0.3$ & $0.4 \pm 0.3$ & $0.0 \pm 0.0$ \\
\hline 13 & 21.10 Unk 91/119/293/371/386 & $0.4 \pm 0.3$ & $0.0 \pm 0.0$ & $0.1 \pm 0.2$ & $0.5 \pm 0.5$ & $0.0 \pm 0.0$ & $0.0 \pm 0.0$ \\
\hline 14 & $21.23 n$-Hexacosane & $0.2 \pm 0.2$ & $0.4 \pm 0.2$ & $0.2 \pm 0.2$ & $0.5 \pm 0.2$ & $0.2 \pm 0.0$ & $0.7 \pm 1.0$ \\
\hline 15 & 21.50 Docosanoic acid & $0.2 \pm 0.1$ & $0.7 \pm 0.3$ & $0.2 \pm 0.2$ & $0.8 \pm 0.2$ & $0.1 \pm 0.2$ & $0.6 \pm 0.6$ \\
\hline 16 & $21.91 n$-Heptacosane & $0.3 \pm 0.2$ & $0.8 \pm 0.3$ & $1.7 \pm 0.6$ & $2.2 \pm 0.4$ & $0.9 \pm 0.4$ & $2.2 \pm 0.5$ \\
\hline 17 & 22.20 Tetracosanol & $0.1 \pm 0.1$ & $0.9 \pm 0.5$ & $0.8 \pm 0.4$ & $1.5 \pm 0.4$ & $<0.1$ & $0.5 \pm 0.4$ \\
\hline 18 & 22.29 Unk 73/179/192/284/311 & $0.0 \pm 0.0$ & $0.0 \pm 0.0$ & $0.0 \pm 0.0$ & $0.0 \pm 0.0$ & $0.0 \pm 0.0$ & $0.0 \pm 0.0$ \\
\hline 19 & $22.66 n$-Octacosane & $0.7 \pm 1.0$ & $0.6 \pm 0.2$ & $0.7 \pm 0.2$ & $1.1 \pm 0.3$ & $1.0 \pm 0.2$ & $1.9 \pm 0.4$ \\
\hline 20 & 22.94 Tetracosanoic acid & $0.1 \pm 0.1$ & $1.0 \pm 0.3$ & $0.4 \pm 0.5$ & $1.6 \pm 0.2$ & $0.4 \pm 0.4$ & $0.7 \pm 0.6$ \\
\hline 21 & 23.04 Unk 73/192/239/432 & $<0.1$ & $0.0 \pm 0.0$ & $0.0 \pm 0.0$ & $0.0 \pm 0.0$ & $0.0 \pm 0.0$ & $0.0 \pm 0.0$ \\
\hline 22 & $23.80 n$-Nonacosane & $3.1 \pm 1.7$ & $3.8 \pm 3.2$ & $19.2 \pm 4.3$ & $9.2 \pm 4.8$ & $43.4 \pm 6.4$ & $37.4 \pm 6.7$ \\
\hline 23 & 24.00 Hexacosanol & $0.6 \pm 0.4$ & $2.3 \pm 0.4$ & $2.4 \pm 1.1$ & $3.0 \pm 0.2$ & $0.1 \pm 0.2$ & $0.0 \pm 0.0$ \\
\hline 24 & $24.51 n$-Triacontane & $1.3 \pm 0.6$ & $0.3 \pm 0.6$ & $0.6 \pm 0.1$ & $0.8 \pm 0.3$ & $1.0 \pm 0.3$ & $1.3 \pm 0.4$ \\
\hline 25 & 24.75 Unk 73/134/224/252/386 & $3.9 \pm 5.1$ & $13.5 \pm 2.0$ & $0.0 \pm 0.0$ & $0.0 \pm 0.0$ & $0.0 \pm 0.0$ & $0.0 \pm 0.0$ \\
\hline 26 & 24.85 Hexacosanoic acid & $0.7 \pm 1.1$ & $0.0 \pm 0.0$ & $1.2 \pm 0.4$ & $2.1 \pm 0.3$ & $0.2 \pm 0.3$ & $1.1 \pm 0.4$ \\
\hline 27 & 25.30 Unk 73/121/134/324/443 & $10.6 \pm 3.3$ & $7.4 \pm 4.3$ & $0.0 \pm 0.0$ & $0.0 \pm 0.0$ & $0.0 \pm 0.0$ & $0.0 \pm 0.0$ \\
\hline 28 & $25.80 n$-Hentriacontane & $1.7 \pm 0.4$ & $5.9 \pm 0.8$ & $5.3 \pm 1.1$ & $3.5 \pm 1.8$ & $5.9 \pm 1.2$ & $4.7 \pm 2.1$ \\
\hline 29 & 26.24 Octacosanol & $0.0 \pm 0.0$ & $3.2 \pm 1.8$ & $2.3 \pm 0.4$ & $3.3 \pm 1.1$ & $0.7 \pm 0.2$ & $0.9 \pm 0.3$ \\
\hline 30 & 26.61 Unk 73/324/443/458 & $0.0 \pm 0.0$ & $1.9 \pm 0.9$ & $0.0 \pm 0.0$ & $0.0 \pm 0.0$ & $0.0 \pm 0.0$ & $0.0 \pm 0.0$ \\
\hline 31 & $26.89 n$-Dotriacontane & $1.1 \pm 0.5$ & $0.3 \pm 0.2$ & $0.1 \pm 0.2$ & $0.3 \pm 0.2$ & $0.6 \pm 0.3$ & $0.1 \pm 0.1$ \\
\hline 32 & 27.45 Octacosanoic acid & $0.6 \pm 0.2$ & $0.7 \pm 0.4$ & $1.3 \pm 0.2$ & $1.6 \pm 0.8$ & $0.4 \pm 0.2$ & $0.5 \pm 0.4$ \\
\hline 33 & 27.66 Unk 57/82/96/418/481 & $0.1 \pm 0.1$ & $0.0 \pm 0.0$ & $0.3 \pm 0.3$ & $2.0 \pm 0.7$ & $0.0 \pm 0.0$ & $0.4 \pm 0.3$ \\
\hline 34 & $28.45 n$-Tritriacontane & $4.6 \pm 2.3$ & $1.0 \pm 0.6$ & $0.0 \pm 0.0$ & $0.3 \pm 0.2$ & $0.0 \pm 0.0$ & $0.1 \pm 0.1$ \\
\hline 35 & 29.20 Triacontanol & $4.3 \pm 1.5$ & $3.2 \pm 1.2$ & $0.9 \pm 0.2$ & $1.5 \pm 0.5$ & $0.8 \pm 0.3$ & $0.5 \pm 0.4$ \\
\hline 36 & 30.10 Unk 69/109/137/411 & $9.2 \pm 2.1$ & $6.8 \pm 1.5$ & $0.0 \pm 0.0$ & $<0.1$ & $0.0 \pm 0.0$ & $0.0 \pm 0.0$ \\
\hline 37 & 30.51 Unk 71/100/155/197 & $0.2 \pm 0.4$ & $0.4 \pm 0.2$ & $0.0 \pm 0.0$ & $3.0 \pm 1.8$ & $0.0 \pm 0.0$ & $0.0 \pm 0.0$ \\
\hline 38 & $31.10 \beta$-Amyrin & $7.2 \pm 4.2$ & $4.3 \pm 0.7$ & $5.7 \pm 0.5$ & $4.6 \pm 1.7$ & $4.8 \pm 1.9$ & $3.1 \pm 0.8$ \\
\hline 39 & 31.30 Triacontanoic acid & $0.0 \pm 0.0$ & $0.0 \pm 0.0$ & $1.3 \pm 0.2$ & $2.1 \pm 1.1$ & $0.5 \pm 0.3$ & $0.0 \pm 0.0$ \\
\hline 40 & 31.65 Unk 73/95/134/218 & $0.9 \pm 0.8$ & $0.5 \pm 0.3$ & $0.0 \pm 0.0$ & $10.0 \pm 2.6$ & $0.0 \pm 0.0$ & $0.0 \pm 0.0$ \\
\hline 41 & $32.00 \alpha$-Amyrin & $12.1 \pm 5.2$ & $3.8 \pm 1.1$ & $15.2 \pm 3.2$ & $10.1 \pm 2.6$ & $8.6 \pm 4.8$ & $4.4 \pm 1.5$ \\
\hline 42 & 33.09 Unk 73/109/203/218/514 & $0.0 \pm 0.0$ & $0.2 \pm 0.2$ & $0.8 \pm 0.6$ & $2.0 \pm 0.8$ & $3.6 \pm 3.0$ & $1.6 \pm 0.1$ \\
\hline 43 & 33.56 Unk 73/134/203/218/393 & $0.1 \pm 0.2$ & $0.0 \pm 0.0$ & $0.0 \pm 0.0$ & $1.3 \pm 0.6$ & $0.0 \pm 0.0$ & $0.0 \pm 0.0$ \\
\hline 44 & 33.80 Dotriacontanol & $1.8 \pm 0.8$ & $2.3 \pm 0.6$ & $1.0 \pm 0.1$ & $1.0 \pm 0.2$ & $1.1 \pm 0.5$ & $0.3 \pm 0.3$ \\
\hline 45 & 34.10 Unk 73/129/216/496 & $0.1 \pm 0.2$ & $0.2 \pm 0.2$ & $0.0 \pm 0.0$ & $1.3 \pm 0.3$ & $1.3 \pm 0.5$ & $0.3 \pm 0.3$ \\
\hline 46 & 34.68 Unk 75/95/237/347/485 & $0.5 \pm 0.6$ & $0.7 \pm 0.8$ & $0.0 \pm 0.0$ & $0.0 \pm 0.0$ & $1.2 \pm 0.5$ & $0.9 \pm 1.2$ \\
\hline 47 & 35.08 Unk 73/129/147/203/496 & $0.0 \pm 0.0$ & $0.0 \pm 0.0$ & $2.5 \pm 0.5$ & $2.8 \pm 1.2$ & $0.0 \pm 0.0$ & $0.3 \pm 0.3$ \\
\hline 48 & 35.44 Unk 73/95/147/175/218 & $1.0 \pm 0.8$ & $1.8 \pm 0.8$ & $0.0 \pm 0.0$ & $0.3 \pm 0.3$ & $0.7 \pm 0.6$ & $0.3 \pm 0.3$ \\
\hline 49 & 36.35 Oleanolic acid & $3.6 \pm 1.3$ & $4.0 \pm 1.2$ & $6.1 \pm 1.8$ & $5.3 \pm 0.3$ & $2.7 \pm 0.8$ & $2.1 \pm 0.8$ \\
\hline 50 & 36.75 Dotriacontanoic acid & $0.3 \pm 0.4$ & $1.3 \pm 1.1$ & $0.0 \pm 0.0$ & $0.8 \pm 0.1$ & $0.0 \pm 0.0$ & $0.1 \pm 0.2$ \\
\hline 51 & 38.50 Ursolic acid & $13.4 \pm 5.1$ & $13.5 \pm 2.6$ & $23.0 \pm 6.0$ & $14.8 \pm 2.4$ & $11.3 \pm 3.5$ & $7.2 \pm 2.2$ \\
\hline 52 & 38.70 Unk 73/133/190/203/320 & $0.1 \pm 0.2$ & $0.5 \pm 0.5$ & $0.9 \pm 1.1$ & $1.3 \pm 0.2$ & $0.6 \pm 0.5$ & $0.3 \pm 0.3$ \\
\hline
\end{tabular}

${ }^{\mathrm{z}} \mathrm{N}$ for each genotype is listed in Table 3 .

yAll unknown components $(<1 \%$ not included) are listed with main mass spectra. 


\begin{tabular}{|c|c|c|c|c|c|c|c|}
\hline \multicolumn{2}{|c|}{$\begin{array}{c}R . \\
\text { viscosum } \\
\text { (susceptible) }\end{array}$} & \multicolumn{2}{|c|}{$\begin{array}{c}R . \\
\text { atlanticum } \\
\text { (susceptible) }\end{array}$} & \multicolumn{2}{|c|}{$\begin{array}{c}R . \\
\text { periclymenoides } \\
\text { (resistant) }\end{array}$} & \multicolumn{2}{|c|}{$\begin{array}{c}R . \\
\text { canescens } \\
\text { (resistant) }\end{array}$} \\
\hline Fall & Spring & Fall & Spring & Fall & Spring & Fall & Spring \\
\hline $0.3 \pm 0.1$ & $1.2 \pm 1.3$ & $0.6 \pm 0.3$ & $1.0 \pm 1.1$ & $0.4 \pm 0.3$ & $1.6 \pm 1.99$ & $0.3 \pm 0.0$ & $1.6 \pm 1.5$ \\
\hline $0.0 \pm 0.0$ & $0.2 \pm 0.2$ & $0.0 \pm 0.0$ & $0.1 \pm 0.1$ & $<0.1$ & $0.1 \pm 0.1$ & $0.0 \pm 0.0$ & $1.1 \pm 1.5$ \\
\hline $0.1 \pm 0.1$ & $0.1 \pm 0.1$ & $0.0 \pm 0.0$ & $0.4 \pm 0.4$ & $0.0 \pm 0.0$ & $0.0 \pm 0.0$ & $0.0 \pm 0.0$ & $0.8 \pm 1.1$ \\
\hline $0.2 \pm 0.3$ & $1.0 \pm 1.0$ & $0.7 \pm 0.8$ & $0.6 \pm 0.3$ & $0.4 \pm 0.4$ & $2.0 \pm 2.1$ & $0.2 \pm 0.2$ & $2.4 \pm 2.4$ \\
\hline $0.3 \pm 0.1$ & $1.1 \pm 1.4$ & $0.7 \pm 0.2$ & $0.7 \pm 0.3$ & $0.2 \pm 0.3$ & $1.0 \pm 1.3$ & $0.2 \pm 0.1$ & $1.8 \pm 1.7$ \\
\hline $0.3 \pm 0.1$ & $0.2 \pm 0.1$ & $0.7 \pm 0.7$ & $3.1 \pm 2.2$ & $0.1 \pm 0.1$ & $0.1 \pm 0.1$ & $0.1 \pm 0.1$ & $1.9 \pm 2.3$ \\
\hline $1.1 \pm 1.4$ & $0.4 \pm 0.3$ & $0.9 \pm 0.4$ & $1.0 \pm 0.2$ & $1.2 \pm 1.7$ & $0.3 \pm 0.0$ & $0.5 \pm 0.5$ & $1.7 \pm 1.9$ \\
\hline $0.5 \pm 0.2$ & $0.4 \pm 0.1$ & $13.0 \pm 10.2$ & $3.2 \pm 1.0$ & $0.2 \pm 0.1$ & $0.2 \pm 0.1$ & $0.3 \pm 0.2$ & $2.6 \pm 2.8$ \\
\hline $0.5 \pm 0.2$ & $0.2 \pm 0.3$ & $0.2 \pm 0.4$ & $1.1 \pm 0.5$ & $0.0 \pm 0.0$ & $0.2 \pm 0.2$ & $0.1 \pm 0.2$ & $1.6 \pm 1.7$ \\
\hline $0.0 \pm 0.0$ & $1.0 \pm 0.2$ & $0.6 \pm 0.4$ & $0.8 \pm 0.8$ & $<0.1$ & $0.3 \pm 0.2$ & $0.0 \pm 0.0$ & $0.0 \pm 0.0$ \\
\hline $1.0 \pm 1.1$ & $0.0 \pm 0.0$ & $0.3 \pm 0.3$ & $0.2 \pm 0.2$ & $0.2 \pm 0.1$ & $0.0 \pm 0.0$ & $0.1 \pm 0.2$ & $1.3 \pm 1.4$ \\
\hline $0.2 \pm 0.0$ & $0.2 \pm 0.1$ & $0.2 \pm 0.3$ & $0.4 \pm 0.3$ & $0.3 \pm 0.1$ & $0.3 \pm 0.1$ & $0.3 \pm 0.2$ & $0.6 \pm 0.5$ \\
\hline $0.1 \pm 0.1$ & $0.5 \pm 0.3$ & $0.8 \pm 0.3$ & $3.7 \pm 1.9$ & $0.2 \pm 0.4$ & $0.3 \pm 0.2$ & $0.2 \pm 0.1$ & $0.2 \pm 0.3$ \\
\hline $0.6 \pm 0.1$ & $1.1 \pm 0.1$ & $1.9 \pm 0.7$ & $3.1 \pm 1.2$ & $1.1 \pm 0.2$ & $0.9 \pm 0.8$ & $1.1 \pm 0.5$ & $1.5 \pm 0.4$ \\
\hline $0.3 \pm 0.2$ & $1.5 \pm 0.3$ & $3.6 \pm 2.5$ & $7.4 \pm 4.3$ & $0.0 \pm 0.0$ & $0.0 \pm 0.0$ & $0.1 \pm 0.2$ & $0.6 \pm 0.3$ \\
\hline $0.0 \pm 0.0$ & $0.0 \pm 0.0$ & $0.0 \pm 0.0$ & $0.0 \pm 0.0$ & $10.9 \pm 8.1$ & $12.0 \pm 6.0$ & $0.0 \pm 0.0$ & $0.0 \pm 0.0$ \\
\hline $0.7 \pm 0.4$ & $1.6 \pm 0.4$ & $0.4 \pm 0.4$ & $0.6 \pm 0.5$ & $0.6 \pm 0.2$ & $1.2 \pm 0.6$ & $0.6 \pm 0.2$ & $0.9 \pm 0.2$ \\
\hline $0.4 \pm 0.3$ & $1.5 \pm 0.7$ & $2.1 \pm 1.2$ & $3.2 \pm 1.0$ & $0.0 \pm 0.0$ & $0.0 \pm 0.0$ & $0.1 \pm 0.2$ & $0.4 \pm 0.3$ \\
\hline $0.0 \pm 0.0$ & $0.0 \pm 0.0$ & $0.0 \pm 0.0$ & $0.0 \pm 0.0$ & $0.1 \pm 0.1$ & $5.7 \pm 1.2$ & $0.0 \pm 0.0$ & $0.0 \pm 0.0$ \\
\hline $27.4 \pm 14.5$ & $31.5 \pm 6.9$ & $5.6 \pm 1.5$ & $3.3 \pm 0.5$ & $23.7 \pm 7.3$ & $16.9 \pm 5.0$ & $26.6 \pm 3.9$ & $12.1 \pm 5.7$ \\
\hline $0.7 \pm 0.2$ & $1.0 \pm 0.7$ & $3.2 \pm 2.0$ & $3.8 \pm 1.1$ & $0.2 \pm 0.1$ & $1.9 \pm 1.9$ & $0.6 \pm 0.3$ & $1.4 \pm 0.5$ \\
\hline $0.6 \pm 0.3$ & $1.1 \pm 0.8$ & $0.0 \pm 0.0$ & $0.2 \pm 0.1$ & $1.0 \pm 0.5$ & $1.3 \pm 0.6$ & $1.0 \pm 0.8$ & $1.0 \pm 0.4$ \\
\hline $0.8 \pm 0.1$ & $2.0 \pm 0.7$ & $1.6 \pm 0.4$ & $1.0 \pm 0.7$ & $0.4 \pm 0.1$ & $1.0 \pm 0.4$ & $0.6 \pm 0.1$ & $1.0 \pm 0.5$ \\
\hline $0.0 \pm 0.0$ & $0.0 \pm 0.0$ & $0.0 \pm 0.0$ & $0.0 \pm 0.0$ & $0.0 \pm 0.0$ & $0.0 \pm 0.0$ & $0.0 \pm 0.0$ & $0.0 \pm 0.0$ \\
\hline $5.7 \pm 2.0$ & $5.8 \pm 1.0$ & $0.1 \pm 0.2$ & $0.3 \pm 0.2$ & $16.5 \pm 2.4$ & $9.1 \pm 5.0$ & $20.5 \pm 3.2$ & $8.1 \pm 3.9$ \\
\hline $2.2 \pm 0.7$ & $3.5 \pm 0.5$ & $2.5 \pm 1.2$ & $1.3 \pm 1.0$ & $0.7 \pm 0.3$ & $2.9 \pm 1.1$ & $1.3 \pm 0.4$ & $2.3 \pm 1.3$ \\
\hline $0.4 \pm 0.1$ & $0.3 \pm 0.2$ & $0.0 \pm 0.0$ & $0.0 \pm 0.0$ & $0.2 \pm 0.4$ & $0.0 \pm 0.0$ & $0.0 \pm 0.0$ & $0.1 \pm 0.1$ \\
\hline $0.0 \pm 0.0$ & $0.1 \pm 0.1$ & $0.1 \pm 0.3$ & $0.6 \pm 0.5$ & $0.4 \pm 0.3$ & $0.3 \pm 0.3$ & $0.2 \pm 0.1$ & $0.3 \pm 0.2$ \\
\hline $1.1 \pm 0.3$ & $1.3 \pm 0.9$ & $1.3 \pm 0.8$ & $0.7 \pm 0.9$ & $0.7 \pm 0.9$ & $0.8 \pm 0.7$ & $0.7 \pm 0.2$ & $0.8 \pm 0.6$ \\
\hline $0.0 \pm 0.0$ & $0.5 \pm 0.4$ & $1.7 \pm 1.4$ & $1.9 \pm 0.8$ & $0.2 \pm 0.2$ & $0.3 \pm 0.4$ & $0.2 \pm 0.2$ & $0.5 \pm 0.5$ \\
\hline $0.0 \pm 0.0$ & $0.4 \pm 0.4$ & $0.0 \pm 0.0$ & $0.0 \pm 0.0$ & $1.0 \pm 1.3$ & $0.6 \pm 0.3$ & $0.7 \pm 0.4$ & $0.7 \pm 0.5$ \\
\hline $2.3 \pm 0.3$ & $2.3 \pm 1.4$ & $3.7 \pm 1.7$ & $1.9 \pm 0.6$ & $0.5 \pm 0.4$ & $1.3 \pm 0.6$ & $0.6 \pm 0.2$ & $0.8 \pm 0.6$ \\
\hline $0.0 \pm 0.0$ & $0.0 \pm 0.0$ & $0.0 \pm 0.0$ & $0.4 \pm 0.3$ & $0.0 \pm 0.0$ & $0.0 \pm 0.0$ & $0.0 \pm 0.0$ & $0.0 \pm 0.0$ \\
\hline $0.0 \pm 0.0$ & $0.0 \pm 0.0$ & $8.1 \pm 9.2$ & $11.7 \pm 2.8$ & $0.0 \pm 0.0$ & $0.0 \pm 0.0$ & $0.0 \pm 0.0$ & $0.0 \pm 0.0$ \\
\hline $7.7 \pm 1.4$ & $4.4 \pm 1.6$ & $2.7 \pm 1.7$ & $2.4 \pm 0.3$ & $0.0 \pm 0.0$ & $0.0 \pm 0.0$ & $0.0 \pm 0.0$ & $1.1 \pm 0.9$ \\
\hline $1.7 \pm 0.8$ & $1.0 \pm 1.0$ & $3.8 \pm 1.7$ & $0.0 \pm 0.0$ & $1.0 \pm 1.4$ & $1.3 \pm 0.2$ & $0.8 \pm 0.3$ & $0.7 \pm 0.6$ \\
\hline $0.0 \pm 0.0$ & $0.0 \pm 0.0$ & $0.0 \pm 0.0$ & $10.4 \pm 9.4$ & $0.0 \pm 0.0$ & $0.0 \pm 0.0$ & $0.0 \pm 0.0$ & $0.1 \pm 0.1$ \\
\hline $18.2 \pm 4.7$ & $6.3 \pm 2.6$ & $13.9 \pm 3.3$ & $3.0 \pm 4.0$ & $1.2 \pm 1.3$ & $0.6 \pm 0.2$ & $1.9 \pm 0.5$ & $2.2 \pm 1.7$ \\
\hline $0.7 \pm 0.9$ & $5.6 \pm 2.9$ & $0.0 \pm 0.0$ & $1.6 \pm 1.1$ & $0.6 \pm 1.3$ & $0.0 \pm 0.0$ & $0.0 \pm 0.0$ & $0.0 \pm 0.0$ \\
\hline $0.0 \pm 0.0$ & $0.0 \pm 0.0$ & $0.0 \pm 0.0$ & $1.5 \pm 1.1$ & $0.4 \pm 0.8$ & $0.0 \pm 0.0$ & $0.0 \pm 0.0$ & $0.0 \pm 0.0$ \\
\hline $0.2 \pm 0.4$ & $0.5 \pm 0.3$ & $0.0 \pm 0.0$ & $0.3 \pm 0.2$ & $1.0 \pm 1.1$ & $0.6 \pm 0.1$ & $0.6 \pm 0.2$ & $0.4 \pm 0.3$ \\
\hline $0.0 \pm 0.0$ & $0.0 \pm 0.0$ & $0.0 \pm 0.0$ & $0.1 \pm 0.2$ & $0.0 \pm 0.0$ & $0.0 \pm 0.0$ & $0.0 \pm 0.0$ & $0.5 \pm 0.3$ \\
\hline $3.0 \pm 1.0$ & $3.9 \pm 2.4$ & $0.0 \pm 0.0$ & $0.0 \pm 0.0$ & $0.0 \pm 0.0$ & $0.0 \pm 0.0$ & $0.0 \pm 0.0$ & $0.0 \pm 0.0$ \\
\hline $0.9 \pm 0.6$ & $0.4 \pm 0.3$ & $1.7 \pm 1.6$ & $1.6 \pm 1.4$ & $0.2 \pm 0.3$ & $0.7 \pm 0.2$ & $1.1 \pm 0.2$ & $0.9 \pm 0.6$ \\
\hline $0.0 \pm 0.0$ & $0.9 \pm 0.6$ & $0.0 \pm 0.0$ & $1.7 \pm 1.1$ & $0.0 \pm 0.0$ & $0.0 \pm 0.0$ & $0.0 \pm 0.0$ & $0.0 \pm 0.0$ \\
\hline $2.7 \pm 0.8$ & $1.5 \pm 0.5$ & $3.0 \pm 0.5$ & $1.1 \pm 0.8$ & $6.1 \pm 1.1$ & $6.1 \pm 0.8$ & $6.7 \pm 0.8$ & $5.3 \pm 3.1$ \\
\hline $0.3 \pm 0.4$ & $0.7 \pm 0.2$ & $0.0 \pm 0.0$ & $0.3 \pm 0.3$ & $0.6 \pm 0.7$ & $0.8 \pm 0.9$ & $<0.1$ & $0.5 \pm 0.8$ \\
\hline $10.9 \pm 3.2$ & $5.4 \pm 1.3$ & $12.1 \pm 5.4$ & $4.9 \pm 1.1$ & $23.4 \pm 6.2$ & $20.9 \pm 0.4$ & $28.6 \pm 4.2$ & $16.6 \pm 7.8$ \\
\hline $1.1 \pm 0.4$ & $0.4 \pm 0.3$ & $0.0 \pm 0.0$ & $0.6 \pm 1.0$ & $0.6 \pm 1.0$ & $0.3 \pm 0.6$ & $0.2 \pm 0.2$ & $0.8 \pm 0.6$ \\
\hline
\end{tabular}


genotype was formerly classified as $R$. serrulatum (Small) Millais, and here will be referred to as $R$. serrulatum. All taxa were 1-yearold plants propagated from cuttings. Plants were transplanted into the field under the shade of mixed deciduous trees in mid-November 1994, watered by drip irrigation as needed, and fertilized twice per year with azalea, camellia, rhododendron fertilizer $11 \mathrm{~N}-5 \mathrm{P}-5 \mathrm{~K}$ (STA-Green Plant Food Company, Inc., Sylacauga, Ala.). No pesticides were used during the experiments.

EPICUTICULAR LIPID EXTRACTION ANALYSIS. Foliage lipid analysis was performed according to the procedures described in Balsdon et al. (1995). Each sample, consisting of five undamaged, mature leaves in the middle strata from each plant, were collected on 25 May and 24 Sept. 1995. Temperature in May averaged $71.7^{\circ} \mathrm{C}$. During September average temperature was $71.0^{\circ} \mathrm{C}$. Five-leaf samples were collected from three to five plants of each taxon (Table 1). These were the same plants used to evaluate resistance to azalea lace bug in laboratory and field tests (Wang et al., 1998). Samples were wrapped in foil and allowed to dry at 22 to $25^{\circ} \mathrm{C}$. Dried leaf samples were immersed in chloroform for 40 to $60 \mathrm{~s}$ at room temperature. Extract volumes were reduced in vacuo at $45^{\circ} \mathrm{C}$, then desiccated under a stream of $\mathrm{N}_{2}$. Extracts were derivatized with $80 \mu \mathrm{L} \mathrm{N}, \mathrm{O}$ bis(trimethylsilyl)acetamide at $110^{\circ} \mathrm{C}$ for $10 \mathrm{~min}$, and redried under $\mathrm{N}_{2}$. The derivatized extracts were dissolved in $80 \mu \mathrm{L}$ of hexane.

Aliquots of lipid extracts were analyzed using combined gas chromatography-mass spectrometry (model 5890/ 5972A, Hewlett Packard, PaloAlto, Calif.) equipped with a 5\% polydimethyl megabore cross linked capillary column (30 m, $0.25 \mathrm{~mm}$ i.d., 0.25$\mu \mathrm{m}$ film thickness) and a packed column injector with helium as the carrier gas. The oven was held at $55^{\circ} \mathrm{C}$ for $3.3 \mathrm{~min}$ after sample injection, then raised to $305^{\circ} \mathrm{C}$ at $15^{\circ} \mathrm{C} / \mathrm{min}$ and maintained at 305 ${ }^{\circ} \mathrm{C}$ for $30 \mathrm{~min}$. Individual lipid components were characterized by their electron impact mass spectra (at $70 \mathrm{eV}$ ), compared with those of standards, and matched by computer search with the National Bureau of Standards Mass Spectral Library (Balsdon et al., 1995; Yang et al., 1993). Lipid composition was described as the percentage of the total cuticular lipids based upon the integration of the total ion chromatograms (Yang et al., 1993). One microliter of the standard, octacosanoic acid methyl ester $\left(50 \mathrm{mg} \cdot \mathrm{mL}^{-1}\right)$, was injected once after every five samples to verify system operations.
Azalea laCe bug ReSPOnSE FaCtors. Response factors included oviposition, egg and nymphal survival, duration of nymphal development, and leaf area injured as had previously been determined for each azalea taxon (Wang et al., 1998). Azalea lace bug response factors and lipid compositions were analyzed using SAS GLM procedure (SAS Institute, Cary, N.C.). An arcsine square root transformation was performed on all percentage values before analysis of variance. All means were compared using a Fisher's protected LSD (Snedecor and Cochran, 1967). The relationships of specific lipid compounds and classes to azalea lace bug response factors were analyzed on a per-plant basis by using Pearson correlation coefficients (SAS). Insect responses to individual plants were used in this analysis. Thus, lace bug performance on a particular plant could be related to the leaf epicuticular lipid composition of that plant. Correlations were based on combined seasonal data.

\section{Results}

EPICUTICUlar LIPID COMPOSITION OF AZALEA Foliage. Composition of epicuticular lipids removed from the foliage of six deciduous and one evergreen azalea taxa varied distinctly (Table 1). The major components of surface lipids in the spring and fall from all genotypes were $n$-alkanes and triterpenoids with ursolic acid (peak 51) and $n$ nonacosane (peak 22) being the most prevalent of the lipids. However, $n$-nonacosane (peak 22), $n$-hentriacontane (peak 28), $\alpha$-amyrin, $\beta$-amyrin (peaks 41 and 38), and ursolic acid (peak 51), generally made up a higher percentage of the total lipids in the fall-sampled leaves than in those sampled in the spring.

As a percentage of the total lipids, the triterpenoids, $\alpha$-amyrin, $\beta$ amyrin (peak 41 and 38), ursolic acid (peak 51), and oleanolic acid (peak 49 ), were common in all susceptible genotypes. The resistant $R$. periclymenoides and $R$. canescens generally had low levels of amyrins or completely lacked them (Table 1). Ursolic acid was lowest in susceptible $R$. atlanticum (4.9\% in spring) and highest in resistant $R$. canescens (28.6\% in fall). The lipid, $n$-nonacosane (peak22), was lowest in susceptible 'Delaware Valley White' (3.1\% in fall) and highest in susceptible $R$. serrulatum (43.4\% in fall). The epicuticular lipids also contained a low percentage of $n$-1-alkanols, the dominant compound being triacontanol (peak 35). Levels of the individual $n$-alkanoic acids

Table 2. Composition(\%) by class of cuticular lipid components of leaves from seven azalea taxa (mean \pm SE) collected in the fall or spring.

\begin{tabular}{|c|c|c|c|c|c|c|}
\hline \multirow[b]{2}{*}{$\begin{array}{l}\text { Genotype } \\
\text { (N) }\end{array}$} & \multirow[b]{2}{*}{ Season } & \multicolumn{5}{|c|}{ Component class } \\
\hline & & $\begin{array}{c}n \text {-Alkanes } \\
\left(\mathrm{C}_{22}-\mathrm{C}_{33}\right) \\
\end{array}$ & $\begin{array}{c}n \text {-1-Alkanols } \\
\left(\mathrm{C}_{18}-\mathrm{C}_{32}\right)\end{array}$ & $\begin{array}{c}n \text {-Alkanoic } \\
\text { acids }\left(\mathrm{C}_{16}-\mathrm{C}_{32}\right)\end{array}$ & Amyrin $^{z}$ & $\begin{array}{c}\text { Oleanolic and } \\
\text { ursolic acids } \\
\end{array}$ \\
\hline \multicolumn{7}{|l|}{ Susceptible } \\
\hline Delaware & Fall (5) & $14.6 \pm 1.9$ & $6.8 \pm 1.0$ & $3.4 \pm 0.6$ & $19.2 \pm 4.0$ & $16.9 \pm 2.8$ \\
\hline Valley White & Spring (5) & $14.0 \pm 1.8$ & $11.9 \pm 1.3$ & $6.6 \pm 1.7$ & $8.1 \pm 0.7$ & $17.4 \pm 1.6$ \\
\hline \multirow[t]{2}{*}{ My Mary } & Fall (4) & $28.9 \pm 2.6$ & $7.5 \pm 0.7$ & $6.7 \pm 0.6$ & $20.9 \pm 1.8$ & $29.1 \pm 3.9$ \\
\hline & Spring (5) & $19.1 \pm 3.4$ & $10.3 \pm 0.4$ & $12.1 \pm 1.2$ & $14.7 \pm 1.9$ & $20.1 \pm 1.1$ \\
\hline \multirow[t]{2}{*}{ R. serrulatum } & Fall (5) & $53.8 \pm 3.2$ & $2.7 \pm 0.5$ & $3.1 \pm 0.9$ & $13.4 \pm 2.9$ & $14.0 \pm 1.9$ \\
\hline & Spring (3) & $50.8 \pm 5.2$ & $2.2 \pm 0.6$ & $8.6 \pm 3.4$ & $7.5 \pm 1.4$ & $9.3 \pm 1.7$ \\
\hline \multirow[t]{2}{*}{ R. viscosum } & Fall (4) & $37.1 \pm 7.8$ & $5.6 \pm 0.6$ & $5.6 \pm 0.6$ & $25.9 \pm 3.0$ & $13.6 \pm 2.0$ \\
\hline & Spring (4) & $43.0 \pm 4.0$ & $8.8 \pm 0.9$ & $10.8 \pm 1.4$ & $10.6 \pm 2.0$ & $6.9 \pm 0.8$ \\
\hline \multirow[t]{2}{*}{ R. atlanticum } & Fall (5) & $11.3 \pm 1.4$ & $13.0 \pm 2.0$ & $25.2 \pm 5.2$ & $16.6 \pm 1.6$ & $15.1 \pm 2.6$ \\
\hline & Spring (4) & $17.9 \pm 3.3$ & $14.9 \pm 2.3$ & $14.8 \pm 1.2$ & $5.4 \pm 1.9$ & $6.0 \pm 0.9$ \\
\hline \multicolumn{7}{|l|}{ Resistant } \\
\hline \multirow[t]{2}{*}{ R. canescens } & Fall (5) & $51.9 \pm 1.8$ & $3.2 \pm 0.5$ & $3.4 \pm 0.3$ & $1.9 \pm 0.2$ & $35.4 \pm 2.2$ \\
\hline & Spring (5) & $30.1 \pm 2.7$ & $6.4 \pm 0.8$ & $13.5 \pm 3.1$ & $3.3 \pm 1.9$ & $22.0 \pm 4.8$ \\
\hline \multirow[t]{2}{*}{ R. periclymenoides } & Fall (5) & $46.1 \pm 3.0$ & $2.3 \pm 0.8$ & $4.3 \pm 1.1$ & $1.2 \pm 0.6$ & $29.6 \pm 3.0$ \\
\hline & Spring (3) & $31.2 \pm 6.7$ & $6.7 \pm 1.9$ & $9.1 \pm 3.0$ & $0.6 \pm 0.1$ & $27.0 \pm 0.6$ \\
\hline
\end{tabular}

$\overline{{ }^{2} \text { Triterpenoids. }}$ 
detected were similar to those of $n$-1-alkanols, with different dominant components in different genotypes, and most of them present at higher levels in the spring than in the fall. Three species-specific unknowns (peaks 25, 27, and 36) in 'Delaware Valley White', eicosanoic acid (peak 9), and two other unknowns (peaks 37 and 40) in susceptible $R$. atlanticum, and another species-specific unknown compound (peak 18) in resistant $R$. periclymenoides were also dominant components in these genotypes (Table 1).

The mean sum of each class of lipid compounds (Table 1) varied among genotypes (Table 2). The proportion of $n$-alkanes in 'Delaware Valley White' was seasonally stable at $14.0 \%$ to $14.6 \%$ of the total surface lipids. Thehighestpercentage of $n$-alkaneswasinsusceptible $R$. serrulatum, and ranged from $50.8 \%$ to $53.8 \%$ (Table 2). The proportion of $n-1$ alkanols ranged from $2.2 \%$ of the total lipids in susceptible $R$. serrulatum to $14.9 \%$ in susceptible $R$. atlanticum, with higher levels in the spring for most of the genotypes. The proportion of $n$-alkanoic acids varied from $3.4 \%$ of the lipids in susceptible 'Delaware Valley White' to $25.2 \%$ in susceptible $R$. atlanticum, with twice the amount in the spring as in the fall for most genotypes (Table 2).

More variation among genotypes was observed for the triterpenoids than for the other lipid classes (Table 2). Amyrins were very low in resistant cultivars and much higher in susceptible cultivars. Ursolic and oleanolic acid, however, were high in resistant cultivars and lower in susceptible cultivars. The triterpenoids $\alpha$ amyrin and $\beta$-amyrin (peaks 41 and 38 ) ranged from $0.6 \%$ of the total lipids in the resistant $R$. periclymenoides to $25.9 \%$ in the susceptible $R$. viscosum, with consistently much lower levels in both seasons in the two most resistant species, $R$. canescens and $R$. periclymenoides. Ursolic acid (peak 51) and oleanolic acid (peak 49) ranged from $6.9 \%$ to $13.6 \%$ in $R$. viscosum to $22.0 \%$ to $35.4 \%$ in $R$. canescens, with higher levels in the fall for all deciduous genotypes, whereas 'Delaware Valley White' produced the same levels of these two compounds in the spring and in the fall (Table 2).

RELATIONSHIPOF EPICUTICULAR LIPIDS TO AZALEA LACE BUG RESPONSES ON AZALEAS. Correlation analysis with life history attributes previously measured (Wang et al., 1998) showed that $n$-alkanes, $n$-1-alkanols, and triterpenoids were significantly associated with azalea lace bug nymphal emergence, nymphal survival, duration of nymphal development, and feeding (injured leaf area) (Table 3). Triterpenoids were also associated with oviposition. The $n$-alkanoic acids had no significant correlations with azalea lace bug responses. The different triterpenoids had very different effects on azalea lace bug resistance. Correlation coefficients relating amyrins to lace bug response were significantly positive, indicating that amyrins (peak 38 and 41) were associated with azalea susceptibility. In contrast, ursolic acid (peak 51) and oleanolic acid (peak 49) had significant negative correlations with azalea lace bug responses on azaleas, indicating that their presence is associated with plant resistance
(Tables 3 and 4). Heptadecanoic acid (peak 3), n-hentriacontane (peak 28), and one unknown (Table 1, peak 18) were significantly negatively correlated with oviposition, eclosion, and nymphal survival; they were positively correlated with duration of development indicating a prolonging of the developmental period (Table 4). Triacontanol (peak 35) and one other unknown (peak 11, Table 2, with major mass spectra 73/119/ 387/402) were significantly positively associated with oviposition, nymphal survival, and feeding; but negatively associated with duration of development. A third unknown (peak 46, Table 2) was positively correlated with azalea lace bug responses on oviposition, percentage emergence from the egg, and nymphal survival. A fourth unknown (peak 48 , Table 2) was also positively correlated with oviposition, nymphal survival, and feeding.

\section{Discussion}

Bloom color (Schultz, 1993), leaf pubescence (Braman and Pendley, 1992; Wang et al., 1998), and leaf moisture (Wang et al., 1998) are not consistently associated with azalea lace bug resistance. The statistical correlation analysis between azalea lace bug response factors on azalea leaves and leaf surface chemistry suggested that leaf wax chemistry is a key factor in differential selection of potential hosts by azalea lace bug. Results of previous bioassays (Wang et al., 1998 ) indicated that the resistance in $R$. canescens and $R$. periclymenoides to azalea lace bug was likely due to antixenosis and antibiosis, according to criteria described by Smith et al. (1994).

The plant surface is one of the most critical junctions influencing host acceptance by insects (Eigenbrode and Espelie, 1995). The epicuticular lipid compositions reported here for six deciduous taxa relate the surface lipid composition of azalea foliage to observed resistance to azalea lace bug. The variations in the composition and ratios of epicuticular lipids may influence herbivorous insect behavior (Eigenbrode and Espelie, 1995; Espelie et al., 1991). The seasonal variation of the surface lipids from azalea foliage may explain some of the seasonal differences in the azalea lace bug susceptibility of azalea genotypes (Braman and Pendley, 1992; Schultz, 1993; Wang et al., 1998). For example, on R. atlanticum, as $\alpha$-amyrin increased from the spring to the fall (Table 2 ) the plants became more susceptible (Wang et al., 1998).

Triterpenoids, the common components of epicuticular lipids in a wide variety of plants (Eigenbrode and Espelie, 1995), are very important in azalea lace bug-azalea interactions. Although $\alpha$ amyrin, $\beta$-amyrin, ursolic acid, and oleanolic acid (peaks 41, 38, 51, and 49) are all prevalently found in azaleas, their effects on azalea lace bug were previously undetermined. Yang et al. (1993) observed that the peanut (Arachis hypogaea L.) species most susceptible to pests had the highest levels of $\alpha$-amyrin and $\beta$-amyrin. Our results

Table 3. Pearson correlation coefficients of azalea lace bug oviposition (egg number) and feeding and egg and nymphal development, with six classes of cuticular lipids of leaves of seven azalea taxa.

\begin{tabular}{|c|c|c|c|c|c|}
\hline $\begin{array}{l}\text { Lipid } \\
\text { class }\end{array}$ & $\begin{array}{c}\text { Egg } \\
\text { no. }\end{array}$ & $\begin{array}{c}\text { Emergence } \\
(\%)\end{array}$ & $\begin{array}{c}\text { Nymph } \\
\text { survival } \\
(\%)\end{array}$ & $\begin{array}{c}\text { Nymph } \\
\text { development } \\
\text { time }^{\mathrm{z}}\end{array}$ & $\begin{array}{l}\text { Injured } \\
\text { leaf } \\
\text { area } \\
\left(\mathrm{mm}^{2}\right)\end{array}$ \\
\hline$\overline{n \text {-Alkanes }\left(\mathrm{C}_{22}-\mathrm{C}_{33}\right)}$ & -0.22 & $-0.36^{*}$ & $-0.44^{* *}$ & $0.47^{* *}$ & $-0.54^{* * *}$ \\
\hline$n$-1-Alkanols $\left(\mathrm{C}_{18}-\mathrm{C}_{32}\right)$ & 0.15 & $0.36^{*}$ & $0.34^{*}$ & $-0.36^{*}$ & $0.50^{* * *}$ \\
\hline$n$-Alkanoic acids $\left(\mathrm{C}_{16}-\mathrm{C}_{32}\right)$ & -0.07 & 0.25 & 0.14 & -0.24 & 0.03 \\
\hline$\alpha$-amyrin and $\beta$-amyrin ${ }^{y}$ & $0.58^{* * *}$ & $0.45^{* *}$ & $0.62^{* * * *}$ & $-0.54^{* * *}$ & $0.65^{* * *}$ \\
\hline Oleanolic and ursolic acids ${ }^{\mathrm{y}}$ & $-0.46^{* * *}$ & $-0.40^{* * *}$ & $-0.50^{* * *}$ & $0.45^{* *}$ & $-0.44^{* *}$ \\
\hline
\end{tabular}

${ }^{\mathrm{z}}$ Days from nymph to adult.

yTriterpenoids.

***,*** Significant at $P \leq 0.05,0.01$, or $0.001(\mathrm{~N}=40)$, respectively. 
Table 4. Pearson correlation coefficients of azalea lace bug oviposition (egg number) and feeding and nymphal emergence, survival, and development with eleven cuticular lipid components of leaves of seven azalea taxa.

\begin{tabular}{|c|c|c|c|c|c|}
\hline $\begin{array}{l}\text { Lipid } \\
\text { component } \\
\text { (peak } \\
\text { no. })^{\mathrm{z}}\end{array}$ & $\begin{array}{c}\text { Egg } \\
\text { no. }\end{array}$ & $\begin{array}{c}\text { Emergence } \\
(\%)\end{array}$ & $\begin{array}{c}\text { Nymph } \\
\text { survival } \\
(\%)\end{array}$ & $\begin{array}{c}\text { Nymph } \\
\text { development } \\
\text { time }^{\mathrm{y}}\end{array}$ & $\begin{array}{c}\text { Injured } \\
\text { leaf } \\
\text { area } \\
\left(\mathrm{mm}^{2}\right)\end{array}$ \\
\hline Heptadecanoic acid (3) & $-0.45^{* *}$ & $-0.50^{* * *}$ & $-0.34^{*}$ & $0.39^{*}$ & $-0.52^{* * * *}$ \\
\hline Unknown (11) & 0.26 & $0.42^{* *}$ & $0.34^{*}$ & $-0.32^{*}$ & $0.47^{* *}$ \\
\hline Unknown (18) & $-0.36^{*}$ & $-0.39^{*}$ & -0.21 & 0.21 & $-0.47^{* *}$ \\
\hline$n$-Hentriacontane (28) & $-0.53^{* * *}$ & $-0.45^{* * *}$ & $-0.63^{* * * *}$ & $0.55^{* * *}$ & $-0.64^{* * *}$ \\
\hline Triacontanol (35) & 0.19 & $0.47^{* *}$ & $0.35^{*}$ & $-0.37^{*}$ & $0.49^{* *}$ \\
\hline$\beta$-Amyrin (38) & $0.61^{* * *}$ & $0.41^{* *}$ & $0.61^{* * * *}$ & $-0.52^{* * * *}$ & $0.62^{* * * *}$ \\
\hline$\alpha$-Amyrin (41) & $0.53^{* * *}$ & $0.44^{* * *}$ & $0.59^{* * * *}$ & $-0.52^{* * * *}$ & $0.63^{* * * *}$ \\
\hline Unknown (46) & $0.36^{*}$ & $0.33^{*}$ & $0.35^{*}$ & -0.28 & 0.29 \\
\hline Unknown (48) & $0.47^{* *}$ & 0.16 & $0.32^{*}$ & -0.24 & $0.44^{* * *}$ \\
\hline Oleanolic acid (49) & $-0.47^{* *}$ & $-0.44^{* *}$ & $-0.54^{* * * *}$ & $0.48^{* *}$ & $-0.45^{* *}$ \\
\hline Ursolic acid (51) & $-0.45^{* *}$ & $-0.35^{*}$ & $-0.48^{* *}$ & $0.44^{* *}$ & $-0.43^{\text {** }}$ \\
\hline
\end{tabular}

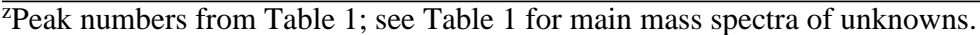

'Days from nymph to adult.

${ }_{*, * *, * * *}$ Significant at $P \leq 0.05,0.01$, or $0.001(\mathrm{~N}=40)$, respectively.

also demonstrated that genotypes susceptible to azalea lace bug had much higher levels of these two components in foliar epicuticular lipids than did resistant genotypes (Tables 1 and 2). These compounds may serve as stimulants for oviposition and/or feeding. However, several studies reported that high levels of these two components were associated with insect resistance (Eigenbrode et al., 1991; Robertson et al., 1991). Our study also suggests that ursolic acid and oleanolic acid (peaks 51 and 49) may have postingestive effects on azalea lace bug because these compounds were significantly positively correlated to nymphal duration of development indicating a prolonged development time (Tables 3 and 4). In addition, feeding injury and oviposition were significantly negatively correlated with ursolic acid and oleanolic acid. Furthermore, the adults that developed from nymphs reared on leaves of genotypes that produce higher levels of these compounds were smaller than individuals reared on other plant taxa.

Heptadecanoic acid $\left(\mathrm{C}_{17}\right)$ contributed to azalea lace bug resistance, perhaps by deterring oviposition and feeding (Table 4). A high level of $n$-hentriacontane $\left(\mathrm{C}_{31}\right)$ was associated with azalea lace bug resistance (Table 4). Mori (1982) found that some components of $n$ alkanols (hexacosanol and octacosanol) stimulated the feeding of silkworm (Bombyx mori L.). In our study, triacontanol (peak 35) was associated with azalea lace bug susceptibility. Several important unknowns were found that may have affected azalea lace bug behavior on azaleas; these need to be further characterized.

In summary, this study suggests that the performance of the azalea lace bug on specific azalea genotypes may be influenced by plant surface chemistry. However, the effects of specific epicuticular lipids need to be further elucidated. Identification of the specific chemical constituents that impart resistance to azalea lace bug will facilitate the development of biochemical assays that can be used to screen resistant progeny in segregating populations, and may provide useful biopesticide information for controlling this insect.

\section{Literature Cited}

Balsdon, J.A., K.E. Espelie, and S.K. Braman. 1995. Epicuticular lipids from azalea (Rhododendron spp.) and their potential role in host plant acceptance by azalea lace bug, Stephanitis pyrioides (Heteroptera: Tingidae). Biochem. Syst. Ecol. 23:477-485.

Bergman, D.K., J.W. Dillwith, A.A. Zarrabi, and R.C. Berberet. 1991. Epicuticular lipids of alfalfa relative to its susceptibility to spotted alfalfa aphids (Homoptera: Aphididae). Environ. Entomol. 20:781-785.
Braman, S.K. and A.F. Pendley. 1992. Evidence for resistance of deciduous azaleas to azalea lace bug. J. Environ. Hort. 10:40-43.

Braman, S.K., A.F. Pendley, B. Sparks, and W.G. Hudson. 1992. Thermal requirements for development, population trends, and parasitism of azalea lace bug (Heteroptera: Tingidae). J. Econ. Entomol. 85:870-877.

Eigenbrode, S.D. and K.E. Espelie. 1995. Effects of plant epicuticular lipids on insect herbivores. Annu. Rev. Entomol. 40:171-194.

Eigenbrode, S.D., K.E. Espelie, and A.M. Shelton. 1991. Behavior of neonate diamondback moth larvae [Plutella xylostella (L.)] on leaves and on extracted leaf waxes of resistant and susceptible cabbages. J. Chem. Ecol. 17:16911704.

Espelie, K.E., E.A. Bernays, and J.J. Brown. 1991. Plant and insect cuticular lipids serve as behavioral cues for insects. Arch. Insect Biochem. Physiol. $17: 223-233$.

Johnson, A.W. and R.F. Severson. 1984. Leaf surface chemistry of tobacco budworm resistant tobacco. J. Agr. Entomol. 1:23-32.

Lowe, H.J.B., G.J.P. Murphy, and M.L. Parker. 1985. Non-glaucousness, a probable aphid-resistance character of wheat. Ann. Appl. Biol. 106:555-560.

Mori, M. 1982. $n$-Hexacosanol and $n$-octacosanol: Feeding stimulants for larvae of the silkworm, Bombyx mori. J. Insect Physiol. 11:969-973.

Neal, J.W. and L.W. Douglass. 1988. Development, oviposition rate, longevity, and voltinism of Stephanitis pyrioides (Heteroptera: Tingidae), an adventive pest of azaleas at three temperatures. Environ. Entomol. 17:827-831.

Raupp, M.J. and R.M. Noland. 1984. Implementing landscape plant management programs in institutional and residential settings. J. Arboricult. 10:161169.

Robertson, G.W., D.W. Griffiths, A.N.E. Birch, A.T. Jones, J.W. McNicol, and J.E. Hall. 1991. Further evidence that resistance in raspberry to the virus vector aphid, Amphorophora idaei, is related to the chemical composition of the leaf surface. Ann. Appl. Biol. 119:443-449.

Schultz, P.B. 1993. Host plant acceptance of azalea lace bug (Heteroptera: Tingidae) for selected azalea cultivars. J. Entomol. Sci. 28:230-235.

Smith, C.M., Z.R. Khan, and M.D. Pathak. 1994. Techniques for evaluating insect resistance in crop plants. CRC Press, Boca Raton, Fla.

Snedecor, G.W. and W.G. Cochran. 1967. Statistical methods. 6th ed. Iowa State Univ. Press, Ames. p. 593.

Starks, K.J. and D.E. Weibel. 1981. Resistance in bloomless and sparse-bloom sorghum to greenbug. J. Econ. Entomol. 10:963-965.

Wang, Y., C.D. Robacker, and S. K. Braman. 1998. Identification of resistance to azalea lace bug among deciduous azalea taxa. J. Amer. Soc. Hort. Sci. 123:592-597.

Yang, G., K.E. Espelie, J.W. Todd, A.K. Culbreath, R.N. Pittman, and J.W. Demski. 1993. Cuticular lipids from wild and cultivated peanuts and the relative resistance of these peanut species to fall armyworm and thrips. J. Agr. Food Chem. 41:814-818. 